\title{
Augmenting a Research Information System with automatically acquired category and keyword information
}

\author{
Sven Blanck, Andreas Niekler and Marc Kaulisch \\ \{marc.kaulisch,sven.blanck\}@zv.uni-leipzig.de,aniekler@informatik.uni-leipzig.de \\ Leipzig University, Ritterstraße 26, 04109 Leipzig (Germany)
}

\begin{abstract}
With an ever-increasing amount of data, it is essential for many systems that documents can be retrieved efficiently. The process of information retrieval can be supported by metadata enrichment of the documents. The aim of this work is to make scientific publications and project descriptions, consisting of titles, abstracts and bibliographical references, easier to find. Therefore, we investigate text analytical methods such as keyword extraction algorithms (TFIDF, Log-Likelihood, RAKE, TAKE and KECNW) and classification approaches using a SVM with ensembles of classifier chains (Web of Science and GEPRIS categories as taxonomies) and compare their quality. We present an altered an optimized keyword extraction algorithm and a supervised subject and keyword classification approach which are, to our knowledge so far, one of the first automatic applications of this kind in informetrics and scientific information retrieval.

The most promising methods are employed and the extracted information is attached to the documents as metadata. These support a search query, using pseudo relevance feedback, to obtain further relevant search results and can also be used to derive profiles for authors, faculties, etc. The concepts developed here will serve as a basis for the Leipzig University Research Information System.
\end{abstract}

\section{Introduction}

In this paper, we present an evaluation of methods used in Natural Language Processing and Text Mining in order to generate unified keywords, classify projects and publications and improve findability of research information. Our aim is to develop a (semi-) automatic annotation approach for research information, which provides a standardized and unified way to augment this information with additional metadata such as keywords and classifications.

The self-developed Leipzig University Research Information System (leuris) targets metadata about projects, publications, prices/awards, international research partnerships, academic events, patents, transfer activities and researcher education. In each information entity, the text data such as titles, abstracts or full-texts are collected. Leipzig University aims to use this text data threefold. First, this text data is analysed in order to profile research at Leipzig University internally. Second, text data analysis improves the findability of research information for external and internal purposes. Third, the utilization of text data enables content-based classification in order to ease further internal analysis and to display annotated research information users of the information system.

Keywords or subject areas are important metadata of research projects, research proposals, conference contributions or journal articles. However, each publisher, funder or conference organizer might use a different subject category system and often leaves the keyword selection decision to the authors. Within a Current Research Information System (CRIS), this leads to a heterogeneous and large set of keywords and subjects in large collections of documents. This ultimately results in ambiguous keywords which make it hard for a CRIS to use such information for performant and effective searches. However, these manually assigned keywords can be used as metadata to optimize a CRIS - not only for search tasks but also for classifying research activities - in addition to automatically extracted keywords and classes.

In the literature there are essentially two different ways to automatically generate keywords from a given text (Beliga, Meštrović, \& Martinčić-Ipšić, 2015). The first type is keyword extraction. This weights the terms that appear directly in the given text according to their importance and sets the most important ones as keywords as they declare the documents main contents. The second approach is keyword assignment. We assess the problem of keyword 
assignment with the automatic classification of given category schemes as it might be difficult or impossible to create, maintain and extend a controlled uniform technical vocabulary for multiple disciplines (Singhal, Kasturi, \& Srivastava, 2013). Therefore, we decided to evaluate individual methods for keyword extraction and keyword assignment. We assess the problem of keyword assignment with the automatic classification of given category schemes.

The novelty of our contribution is the alteration of known approaches to fit scientific publications or scientific texts. In detail, we changed term weighting schemes and score aggregation functions of the underlying algorithms and evaluated their results in order to fit the methods to the here described text sources. Additionally, we present a supervised subject and keyword classification approach which is, to our knowledge so far, one of the first applications of this kind in informetrics and scientific information retrieval.

\section{Related Work}

In this paper we present a Text Mining-based approach to extract full-text-based metainformation. This information will later be used in information retrieval tasks within leuris. Information retrieval and informetrics are symbiotic fields (Wolfram, 2015). In scientometrics/informetrics fields text analysis and information retrieval is among other things used for topic clustering and finding possible specialities (Sjögårde \& Ahlgren, 2019).

For some time now, statistical methods of keyword extraction have offered the best results with regard to precision, recall and F1 score. Approaches such as TF-IDF (Sparck Jones, 1972) and Log-Likelihood (LL) (Rayson, Berridge, \& Francis, 2004) use a reference corpus to rank individual terms. Especially the TF-IDF measure is popular as a baseline to compare and evaluate new algorithms. Up to this point, it is the workhorse in many applications such as the Solr/Lucene full-text index.

Another group of keyword extraction approaches is graph-based. As stated by Beliga et al. Graph-based approaches represent a good alternative to statistical approaches due to their independence from linguistic knowledge, domain and language (Beliga et al., 2015). Part of this work is aimed at evaluating the results of statistical methods with graph-based methods such as RAKE (Rose, Engel, Cramer, \& Cowley, 2010), TAKE (Pay, 2016) and KECNW (keyword extraction using collective node weight) (Biswas, Bordoloi, \& Shreya, 2017), which is a further development on TextRank (Mihalcea \& Tarau, 2004) and NE-Rank (Bellaachia \& Al-Dhelaan, 2012). While statistical and graph-based methods arrange the individual terms according to their relevance, RAKE and TAKE also employ methods for multi-term keyword candidate recognition using linguistic knowledge (Hulth, 2003). We apply the strategies, which create multi-term keyword candidates to the other approaches (KECNW, TF-IDF, LL) as well. Our second approach, a supervised classification of scientific categories, has originally been described in (Brück, Eger, \& Mehler, 2016; Uslu, Mehler, Niekler, \& Baumartz, 2018; Waltinger, Mehler, Lösch, \& Horstmann, 2009). In this work, the authors describe a classification mechanism using the word contents of short text snippets in order to assign a DDC category to each of the short texts. The authors utilize a Support Vector Machine (SVM) to classify the text snippets. Uslu et al. (2018) extend this approach by combining a SVM and a Neural Network Classifier with only a slight performance gain and we therefore stick to the original approach and will use only SVM classification for ease of understanding. However, a SVM is normally only responsible for assigning one label to a text. In our case, we need to assign multiple labels to a single document that will act as categories in later applications. One problem transformation that has proven to be practical is One-vs-Rest classification also referred to as binary relevance (BR) (Aly, 2005). Label relations amongst each other can be integrated into the learning process of a SVM with the help of classifier chains (CC) (Read, Pfahringer, Holmes, \& Frank, 2009). A further improvement are ensembles of classifier chains 
(ECC), which minimize the risk of a poorly chosen classification order through multiple iterations of CC and majority voting (Read, Pfahringer, Holmes, \& Frank, 2011).

In our work, we use the extracted metadata consisting of keywords and categories to expand search queries and hence improve the performance of retrieval systems. As Xu \& Croft (1996) confirmed in their experiments, an expansion with local feedback based on the search results of the original query is more suitable than global techniques that examine word relationships in a corpus. Therefore, the application examples in this paper use an expansion with local feedback called Pseudo Relevance Feedback (PRF), as this technique currently represents the state of the art (Ariannezhad, Montazeralghaem, Zamani, \& Shakery, 2017; Keikha, Ensan, \& Bagheri, 2017).

\section{Datasets}

In this paper, we are investigating methods to extract keywords and classify research information into suitable classifications on the basis of scientific abstracts. In order to do so, we need to utilize different datasets for training and testing. We used a suited dataset to evaluate the keyword extraction methods. For this purpose, we acquired a dataset from the Web of Science, which contains examples of the category Computer Science, Interdisciplinary Applications in order to design and evaluate the keyword extraction algorithms. Based on the assumption that there is a good chance to get meaningful keywords in the field of computer science the decision fell on this specific category. Each example in this dataset contains the title, abstract, references and a set of keywords chosen by the authors. Since the presented methods can only find keywords that are directly in the title or abstract, all author keywords that are not directly contained in the title or abstract are ignored for further evaluation. In order to avoid filtering out different inflections the titles, abstracts, bibliographical references and authors keywords where lemmatized. We use only publications with more than three keywords and out of the 1056 initial examples, 603 remain for our evaluation purposes.

We chose datasets from the Web of Science and the German Project Information System ${ }^{1}$ to create and evaluate subject classification models. For the evaluation of the project abstract classification in German, the GEPRIS taxonomy ${ }^{2}$ was used, which consists of four granularity levels. We had no access to a dataset containing the fourth level. Hence, only the first three levels where used in the category classification experiments. The GEPRIS dataset consists of the title, the project description and the corresponding category from the GEPRIS taxonomy. The datasets divide into the three levels as follows:

- First Level: 46547 examples, 4 categories

- Second Level: 46547 examples, 14 categories

- Third Level: 28712 examples, 48 categories

We used the Web of Science taxonomy ${ }^{3}$ for the evaluation of publication abstract classification in English. To assign proper categories to the acquired dataset a publication automatically gets a category assigned depending on the journal in which it was originally published. Additionally, the CWTS Leiden main fields ${ }^{4}$ provide broader topics and can easily be mapped from the Web of Science categories. This works for all but three of the Web of Science categories, which have no mapping yet. Each of the examples contained in the dataset consists of the title, the abstract, the bibliography and the corresponding category from either

\footnotetext{
${ }^{1}$ GEPRIS, http://gepris.dfg.de/gepris/OCTOPUS?language=en

${ }^{2}$ GEPRIS taxonomy, http://www.dfg.de/dfg_profil/gremien/fachkollegien/faecher/index.jsp

${ }^{3}$ Web of Science taxonomy,

https://images.webofknowledge.com/images/help/WOS/hp_subject_category_terms_tasca.html

${ }^{4}$ Leiden main fields, http://www.leidenranking.com/information/fields
} 
the Web of Science or the CWTS Leiden taxonomy.

We divided the dataset as follows:

- Web of Science categories: 44866 examples, 254 categories

- CWTS Leiden main fields: 42110 examples, 5 categories

As a last step we applied our evaluated methods to different datasets based on publication and project abstracts derived from the Leipzig University Research Report ${ }^{5}$ and inserted the results into a SOLR index.

\section{Keyword and Phrase Detection}

In general, all keyword extraction algorithms presented in the related work use a score calculation for the individual terms (unigrams) to determine their relevancy within the document. RAKE and TAKE additionally use methods to extract multi-term keyword candidates from the text and rank them by using the sum of the individual term scores. We assume that the presented keyword extraction methods can be divided into three sections:

1. Keyword candidate extraction

2. Score calculation of the individual terms

3. Ranking of the keyword candidates

\section{Keyword candidate extraction}

The approaches from RAKE and TAKE were used for the candidate extraction. In short, RAKE uses a stopword list to divide a text into word sequences and uses them as candidates. The original TAKE approach uses Part-of-Speech Tags to extract noun chunks with eventually colocated adjectives from the text. This approach is based on Hulth's studies which show that linguistic knowledge with noun chunks achieves better results in a keyword extraction process than the simple use of n-grams (Hulth, 2003). Our program extracts the candidates from the title and the abstract, which are combined into one single text using a full stop as sentence separator. The bibliographical references will be used later to calculate the individual term scores. SpaCy (Honnibal \& Montani, 2017) is used as a POS tagger and lemmatizer for TAKE and we used a stopword list as a separation feature for RAKE.

\section{Score calculation of individual terms}

To calculate the score we tested several methods. These include the statistical approaches TFIDF and the Log-Likelihood-Ratio. We also tested the graph-based methods KECNW and RAKE respectively TAKE, which are both based on the same principle. In addition, the evaluation includes a combination of TF-IDF and KECNW, which will be discussed later.

In TF-IDF, the individual words are arranged in descending order according to their relevance and the n-best are used as keywords. Note, the IDF value is obtained from the reference corpus. The Log-Likelihood-Ratio uses two corpora to calculate the keywords. The first corpus is the corpus to be examined and the second serves as the reference corpus. Basically, the method carries out a statistical significance test if a selected keyword differs from its statistical expectation. The Log-Likelihood-Method was developed based on the Chi-Squared-Test. The Chi-Squared-Test is based on a normal distribution for statistical text analysis. As an alternative for short texts Dunning suggests the Log-Likelihood-Ratio (Dunning, 1993). This ratio is not based on the assumption that statistical textual analysis is a normal distribution and assumes a binomial and/or multinomial distribution. We simply calculate the RAKE / TAKE (RaTa) based score by the degree of a word divided by its occurrence frequency within a document. The degree is the number of co-occurrences with other words within a specified window in a single document. In the evaluation, a window size of 2 was chosen. KECNW is another algorithm for

\footnotetext{
${ }^{5}$ Leipzig University Research Report, https://fob.uni-leipzig.de/\#/welcome
} 
the automatic extraction of keywords, which was developed especially for Twitter data and based on the ideas of PageRank and TextRank. KECNW is divided into four phases: Textual pre-processing, building a graph structure, node weight assignment and keyword extraction. Each token in the texts will be become a node in the resulting graph structure. An additional filtering is applied by deleting all words which do not exceed the Average Occurrence Frequency (AOF). The edges are formed at words that directly adjoin each other in a sequence. The weighting of an edge is determined by the co-occurrence frequency of a term $i$ and a term $j$, as well as their individual frequencies. In the next step, the nodes are weighted. The weighting is composed of five different components: Distance to the central node $\left(D_{C(i)}\right)$, selectivity centrality $(S C(i))$, importance of neighbouring nodes $\left(N e i g h_{I m p}(i)\right)$, position of a node $(F(i), L(i))$ and term frequency $(T F(i))$. These values are normalized for further calculation. For details on the formulas please refer to the original paper (Biswas et al., 2017). We alter the formulas later but leave out the calculation details for reasons of compactness. Since KECNW is designed for the analysis of Twitter texts (tweets, very short), it is necessary to alter the algorithm for scientific texts. In detail, we exchanged the pre-processing and the additional weighting of the first and last word in the text. We simply, in the case of scientific texts, weighted the first and last words from the title and abstract $(F(i), L(i))$. An additional weighting of the first and last words in the literature references was omitted, since these have only a supporting function and should not influence the result excessively. In addition, we experimented with several parameters. The AOF which must be used according to the original KECNW algorithm is ignored because the abstracts are short texts and possibly important words, which occur only once, are omitted. As our experiments confirmed, omitting the AOF did indeed improve the results for our short documents.

The TF-IDF and the Log-Likelihood-ratio computation needed a comparison corpus. We choose a collection of 1 million sentences of the English Wikipedia of the year 2016 made available by a project within Leipzig University (Uwe Quasthoff \& Eckart, 2015). These sentences are lemmatized and brought into a bag-of-word representation.

In addition to the normal KECNW approach, the following modifications were tested:

- $K E C N W$-Trigram does not only use the direct neighbours, but also the neighbours of the neighbours as edges.

- KECNW-Sentence uses all words that occur in the same sentence as edges.

- KECNW_multiply_idf additionally uses the IDF for the node weighting. The modified node weighting function looks as follows:

$$
\text { Node_weight }(i)=\left(D_{C(i)}+S C(i)+N e i g h_{I m p}(i)+F(i)+L(i)+T F(i)\right) \cdot \operatorname{IDF}(\mathrm{i})
$$

- KECNW_use_tfidf also uses the IDF from the TF-IDF weighting scheme. The modified node weighting function then looks as follows:

$$
\text { Node_weight }(i)=D_{C(i)}+S C(i)+N e i g h_{I m p}(i)+F(i)+L(i)+(T F(i) \cdot \operatorname{IDF}(\mathrm{i}))
$$

All these modifications slightly improved the results as our evaluation demonstrates. The best modification proves to be $K E C N W \_$use_tfidf. However, the quality gain is only in the range 0.05 around the F1 score results of the TF-IDF keyword extraction approach.

\section{Ranking of the keyword candidates}

The original approach of candidate scoring uses the sum of all scores of the individual terms belonging to a keyword candidate. Note, multiple words can form a longer sequence for a keyword candidate. However, this approach favours much longer candidates because the sum gets larger with more words in a candidate sequence. This is undesirable and alternatively, we used the average of the individual terms instead of the sum. As confirmed by our evaluation, this method achieves better results and is therefore used in our implementations. 


\section{Evaluation}

The evaluation measures for these approaches are precision, recall and the F1 score. The keywords are tested with the specified keyword dataset from the Web of Science.

We evaluated scores for increasing numbers $n$ of most relevant keywords detected by the algorithms. The results are shown in Table 1, Table 2 and Table 3. Since most authors assign multi-term keywords, each automatically extracted keyword that is present in the authors keywords only partially is counted as a hit. It is noticeable that the TF-IDF, Log-Likelihood and KECNW are very close to each other. Only RAKE/TAKE (RaTa) is far behind in comparison.

To test the average scores for multi-word keywords we extracted the candidates with the TAKE methodology (RAKE candidates were found to be not optimal). The evaluation is presented in Table 4, Table 5 and Table 6 and demonstrates that the quality of the scoring approaches is similar to the single word extraction evaluation, but a candidate only counts as a hit if the extracted keyword is completely matching an author's keyword. We used the variant $K E C N W$ use_idf for testing the KECNW algorithm, since it turned out it slightly improves the results.

Table 1: Precision of the original individual terms scoring algorithms with partial hits

\begin{tabular}{l|llllllllll}
\hline Method & $n=1$ & $n=2$ & $n=3$ & $n=4$ & $n=5$ & $n=6$ & $n=7$ & $n=8$ & $n=9$ & $n=10$ \\
\hline TF-IDF & $\mathbf{0 . 6 2}$ & 0.56 & $\mathbf{0 . 5 1 8}$ & $\mathbf{0 . 4 7}$ & $\mathbf{0 . 4 3}$ & $\mathbf{0 . 3 9 6}$ & $\mathbf{0 . 3 6 6}$ & $\mathbf{0 . 3 4 3}$ & $\mathbf{0 . 3 2 1}$ & $\mathbf{0 . 3 0 2}$ \\
LL & $\mathbf{0 . 6 2}$ & $\mathbf{0 . 5 6 7}$ & $\mathbf{0 . 5 1 8}$ & 0.469 & $\mathbf{0 . 4 3}$ & 0.394 & 0.365 & 0.341 & 0.32 & 0.3 \\
RaTa & 0.011 & 0.01 & 0.011 & 0.012 & 0.011 & 0.011 & 0.013 & 0.014 & 0.015 & 0.017 \\
KECNW & 0.605 & 0.558 & 0.51 & 0.464 & 0.421 & 0.391 & 0.36 & 0.335 & 0.312 & 0.295 \\
\hline
\end{tabular}

Table 2: Recall of the original individual terms scoring algorithms with partial hits

\begin{tabular}{l|llllllllll}
\hline Method & $n=1$ & $n=2$ & $n=3$ & $n=4$ & $n=5$ & $n=6$ & $n=7$ & $n=8$ & $n=9$ & $n=10$ \\
\hline TF-IDF & $\mathbf{0 . 1 2 3}$ & 0.222 & $\mathbf{0 . 3 0 8}$ & $\mathbf{0 . 3 7 2}$ & $\mathbf{0 . 4 2 6}$ & $\mathbf{0 . 4 7}$ & $\mathbf{0 . 5 0 7}$ & $\mathbf{0 . 5 4 3}$ & $\mathbf{0 . 5 7 1}$ & $\mathbf{0 . 5 9 8}$ \\
LL & $\mathbf{0 . 1 2 3}$ & $\mathbf{0 . 2 2 4}$ & 0.307 & $\mathbf{0 . 3 7 2}$ & 0.425 & 0.468 & 0.505 & 0.539 & 0.57 & 0.594 \\
RaTa & 0.002 & 0.004 & 0.007 & 0.009 & 0.011 & 0.014 & 0.018 & 0.022 & 0.027 & 0.033 \\
KECNW & 0.12 & 0.221 & 0.303 & 0.368 & 0.417 & 0.464 & 0.498 & 0.531 & 0.556 & 0.584 \\
\hline
\end{tabular}

Table 3: F1 score of the original terms scoring algorithms with partial hits

\begin{tabular}{l|llllllllll}
\hline Method & $n=1$ & $n=2$ & $n=3$ & $n=4$ & $n=5$ & $n=6$ & $n=7$ & $n=8$ & $n=9$ & $n=10$ \\
\hline TF-IDF & $\mathbf{0 . 2 0 5}$ & 0.318 & $\mathbf{0 . 3 8 6}$ & $\mathbf{0 . 4 1 5}$ & $\mathbf{0 . 4 2 8}$ & 0.43 & $\mathbf{0 . 4 2 5}$ & $\mathbf{0 . 4 2 1}$ & $\mathbf{0 . 4 1 1}$ & $\mathbf{0 . 4 0 2}$ \\
LL & $\mathbf{0 . 2 0 5}$ & $\mathbf{0 . 3 2 2}$ & $\mathbf{0 . 3 8 6}$ & $\mathbf{0 . 4 1 5}$ & 0.427 & $\mathbf{0 . 4 2 8}$ & 0.424 & 0.418 & 0.41 & 0.399 \\
RaTa & 0.004 & 0.006 & 0.008 & 0.01 & 0.011 & 0.012 & 0.015 & 0.017 & 0.02 & 0.022 \\
KECNW & 0.2 & 0.316 & 0.38 & 0.41 & 0.419 & 0.425 & 0.418 & 0.411 & 0.4 & 0.392 \\
\hline
\end{tabular}

Table 4: Precision of the keywords using TAKE candidates by means of average and exact matching

\begin{tabular}{l|rllllllllr}
\hline Method & $n=1$ & $n=2$ & $n=3$ & $n=4$ & $n=5$ & $n=6$ & $n=7$ & $n=8$ & $n=9$ & $n=10$ \\
\hline TF-IDF & 0.222 & 0.198 & $\mathbf{0 . 1 6 9}$ & 0.149 & 0.138 & $\mathbf{0 . 1 3 0}$ & $\mathbf{0 . 1 2 3}$ & 0.115 & $\mathbf{0 . 1 1 1}$ & $\mathbf{0 . 1 0 5}$ \\
LL & 0.221 & 0.194 & 0.168 & 0.149 & 0.138 & 0.128 & 0.120 & 0.115 & 0.110 & 0.105 \\
RaTa & 0.054 & 0.060 & 0.067 & 0.067 & 0.067 & 0.065 & 0.064 & 0.064 & 0.063 & 0.063 \\
KECNW & $\mathbf{0 . 2 3 3}$ & $\mathbf{0 . 1 9 9}$ & $\mathbf{0 . 1 6 9}$ & $\mathbf{0 . 1 5 2}$ & $\mathbf{0 . 1 4 0}$ & $\mathbf{0 . 1 3 0}$ & $\mathbf{0 . 1 2 3}$ & $\mathbf{0 . 1 1 6}$ & 0.110 & $\mathbf{0 . 1 0 5}$ \\
\hline
\end{tabular}


Table 5: Recall of the keywords using TAKE candidates by means of average and exact matching

\begin{tabular}{l|rllllllllr}
\hline Method & $n=1$ & $n=2$ & $n=3$ & $n=4$ & $n=5$ & $n=6$ & $n=7$ & $n=8$ & $n=9$ & $n=10$ \\
\hline TF-IDF & 0.044 & 0.078 & $\mathbf{0 . 1 0 0}$ & 0.118 & 0.137 & $\mathbf{0 . 1 5 4}$ & $\mathbf{0 . 1 7 0}$ & 0.183 & $\mathbf{0 . 1 9 7}$ & $\mathbf{0 . 2 0 8}$ \\
LL & 0.044 & 0.077 & $\mathbf{0 . 1 0 0}$ & 0.118 & 0.137 & 0.152 & 0.167 & 0.182 & 0.195 & 0.207 \\
RaTa & 0.011 & 0.024 & 0.040 & 0.053 & 0.066 & 0.078 & 0.089 & 0.101 & 0.112 & 0.124 \\
KECNW & $\mathbf{0 . 0 4 6}$ & $\mathbf{0 . 0 7 9}$ & $\mathbf{0 . 1 0 0}$ & $\mathbf{0 . 1 2 0}$ & $\mathbf{0 . 1 3 9}$ & $\mathbf{0 . 1 5 4}$ & $\mathbf{0 . 1 7 0}$ & $\mathbf{0 . 1 8 4}$ & 0.196 & 0.207 \\
\hline
\end{tabular}

Table 6: F1 score of the keywords using TAKE candidates by means of average and exact matching

\begin{tabular}{l|rrrrrrrrrr}
\hline Method & $n=1$ & $n=2$ & $n=3$ & $n=4$ & $n=5$ & $n=6$ & $n=7$ & $n=8$ & $n=9$ & $n=10$ \\
\hline TF-IDF & 0.073 & 0.112 & $\mathbf{0 . 1 2 6}$ & 0.132 & 0.138 & $\mathbf{0 . 1 4 1}$ & 0.142 & 0.141 & $\mathbf{0 . 1 4 2}$ & $\mathbf{0 . 1 4 0}$ \\
LL & 0.073 & 0.110 & 0.125 & 0.132 & 0.137 & 0.139 & 0.140 & 0.141 & 0.140 & 0.139 \\
RaTa & 0.018 & 0.034 & 0.050 & 0.059 & 0.066 & 0.071 & 0.075 & 0.078 & 0.081 & 0.083 \\
KECNW & $\mathbf{0 . 0 7 7}$ & $\mathbf{0 . 1 1 3}$ & $\mathbf{0 . 1 2 6}$ & $\mathbf{0 . 1 3 4}$ & $\mathbf{0 . 1 4 0}$ & $\mathbf{0 . 1 4 1}$ & $\mathbf{0 . 1 4 3}$ & $\mathbf{0 . 1 4 3}$ & 0.141 & 0.139 \\
\hline
\end{tabular}

\section{Subject area classification}

To create and evaluate the classifiers, each dataset for the Web of Science and GEPRIS classification is divided into a training (90\%) and a test set (10\%). All publication and project abstracts are pre-processed. This includes lemmatization for English texts and stemming for German ones because a simple lemmatization for German texts was not available in our processing software. We extracted both, unigrams and bigrams, in order to keep a textual context in the feature set for the classifier. We used LIBLINEAR as SVM implementation (Fan, Chang, Hsieh, Wang, \& Lin, 2008). Since the examples can have multiple labels, the problem was transformed to binary relevance classification. Thus, the SVM creates a separate classifier for each label, which only specifies for this label whether it applies to a given document or not. Furthermore, we extended the classification scheme to classifier chains which use the results of the previous classifications for each classifier to take label relationships into account (Read et al., 2011). Classifier Chains (CC) are an extension of the binary relevance method and integrate label relationships into the calculation of classifiers. Both use one model per label for classification. In CC, this is used to form a chain of models. The basic structure of the input vector remains the same, but starting with the second model in the chain, the previous labels are appended to the feature vector. Ensemble of Classifier Chains (ECC) solve the problem of order. To ensure that results do not depend on the order of the classifiers, a constant number of CC classifiers with randomly selected sequences are calculated. Our ensembles of classifier chains use a total of 11 iterations with randomly selected orders of classifiers. In addition, we tested whether it makes sense to change the threshold value for setting the labels. The original method calculates a threshold value by using the mean value of the estimates (either 0 or 1 ) for each classifier of the same label (Method A). For example, when looking at 11 iterations representing 6 predictions indicating 1 and the remaining 5 predictions indicating 0 , the threshold should be less than 6/11. Another proposed approach uses the probability of a classifier that the label should be set (between 0 and 1) instead of the exact estimate (Method B). The latter methodology can considerably improve the results as shown in Table 7 and therefore a threshold value based on probabilities was used for all further evaluations. 
Table 7: Comparison of using the traditional and altered threshold calculation scheme while employing ECC.

\begin{tabular}{l|lllll}
\hline & & 0/1 Loss & $\begin{array}{l}\text { Hamming } \\
\text { Loss }\end{array}$ & Accuracy & F1 Score \\
& & & 0.045 & 0.911 & 0.913 \\
Method A & GEPRIS 1 & 0.096 & 0.035 & 0.754 & 0.766 \\
& GEPRIS 2 & 0.279 & 0.02 & 0.591 & 0.597 \\
\hline \multirow{3}{*}{ Method B } & GEPRIS 3 & 0.423 & 0.044 & 0.912 & 0.915 \\
& GEPRIS 1 & 0.097 & 0.027 & 0.813 & 0.828 \\
& GEPRIS 2 & 0.233 & 0.033 & 0.619 & 0.686 \\
\hline
\end{tabular}

In addition, the influence of using over- and undersampling were examined in the case of the Web of Science categories. Oversampling replicates the examples of the minority class until the minority and the majority class have the same size. Undersampling deletes random examples of the majority class until the minority and majority class have the same size. Our evaluation, as shown in Table 8, revealed that oversampling is more suitable for our purposes than undersampling with different tested ratios. Note, the quality differences between Table 7 and Table 8 are due to the different datasets. We simply demonstrate the impact of threshold selection and over- and undersampling. We present the final scores in the next section.

Table 8: Comparison of using Oversampling and Undersampling with different ratios (Minority:Majority) while employing ECC. Note, the Web of Science dataset was used for this evaluation.

\begin{tabular}{l|llll}
\hline & 0/1 Loss & $\begin{array}{l}\text { Hamming } \\
\text { Loss }\end{array}$ & Accuracy & F1 Score \\
\hline Undersampling 1:1 & 0.788 & 0.007 & 0.269 & 0.292 \\
Undersampling 1:2 & 0.786 & 0.007 & 0.271 & 0.289 \\
Oversampling 1:1 & 0.79 & 0.008 & 0.367 & 0.431 \\
\hline
\end{tabular}

\section{Evaluation}

We applied evaluation measures as presented by Read et al (2011) for multilabel classification and the corresponding results are shown in Table 9. The experiments with ECC instead of single classifiers significantly increased the quality depending on the number of different labels. The higher the number of different labels, the more the quality improved. Therefore, all classifiers for the evaluation on Table 9 used ECC. In some examples of the test dataset it may happen that the all probabilities for assigning a label are below the required threshold. In such cases the label with the highest probability is selected for these examples. As expected, the classification quality decreases if more different labels are classified. Nevertheless, the results are sufficiently good and suitable to be used. We also observed misclassified categories, which are nonetheless often semantically similar to the correct labels.

Table 9: Evaluation scores for classification with SVM based on two different datasets and different amounts of categories. The number of categories is noted in brackets.

\begin{tabular}{|l|lll|ll|}
\hline & \multicolumn{3}{|c|}{ GEPRIS } & \multicolumn{2}{c|}{ Web of Science } \\
\cline { 2 - 6 } & First & Level & Second & Third & Normal \\
& $(4)$ & Level (14) & Level (48) & CWTS (5) & \\
\hline 0 / 1 Loss & 0.097 & 0.233 & 0.539 & 0.7 & 0.216 \\
Hamming Loss & 0.044 & 0.027 & 0.033 & 0.008 & 0.055 \\
Accuracy & 0.912 & 0.813 & 0.619 & 0.473 & 0.871 \\
F1 Score & 0.915 & 0.828 & 0.686 & 0.534 & 0.9 \\
\hline
\end{tabular}




\section{Application in Search Index}

We applied our approach for keyword and category extraction on a sample of 4362 unlabelled and unprocessed publications from the Research Report of Leipzig University as described in the Datasets. Of these, we inserted the publication title, abstract, publication year and the first mentioned author into a Solr full-text index. Additionally, we automatically annotated the Web of Science categories, research areas, CWTS Leiden categories and the top 10 keywords per document using our proposed methods. The categories were extracted using ECC and the keywords were determined by using KECNW with the TAKE candidates to represent multiterm keywords. The categories were extracted using ECC and the keywords were determined by using KECNW with the TAKE candidates to represent multi-term keywords.

We illustrate the application in a search index with English examples. The usage examples of the generated metadata are divided into two areas:

1. Expansion of conventional search queries using pseudo relevance feedback $(\mathrm{Xu} \&$ Croft, 1996)

2. Facetted search queries on metadata for analysis purposes

A conventional search query refers to a search within the title and abstracts of the texts with one or more search terms defined by the user. In the full-text index, we defined the individual components such as title and abstract as searchable fields in tokenized form. The query is built with a logical disjunction. Since we assume that a search result has a higher relevance if the search term appears in the title and not only in the abstract, we weighted the search differently. Boosting is used to append a multiplier to single search fields or search terms. We use this mechanism in order to calculate the relevance score. ${ }^{6}$ The search query is expanded by using the results of the conventional search query. We acquire the keywords and Web of Science categories from each of the search results and reintegrate them into the search query. ${ }^{7}$ For example, we find 33 documents when searching for the term cardiology within titles and abstracts. From these the keywords and the categories are extracted, which are used to expand the conventional search with logical disjunctions. In this example, a keyword or a category must appear more than twice in the result set to be embedded into the expanded search query. In addition, we define a boosting factor to each field. It is assumed that search terms, which are included in the title or the abstract of the documents, give more relevance to them. The keywords are also more important for the search than the categories and therefore have a higher boosting factor. The boosting factors are intuitively set as following:

- Title $^{\wedge} 4$

- Abstract $^{\wedge} 2$

- Tokenized Keywords ${ }^{\wedge} 1$

- Untokenized Keywords $\wedge 1.5$

- Categories ${ }^{\wedge} 0.5$

Figure 1 shows how the search query can be expanded directly on a keyword search in the title and abstract. The simple search finds only two categories occurring in documents which contain the query terms. But the documents containing the term adipose tissue also deliver 11 further keywords, which co-occur at least in 2 documents from the search result. These keywords expand the search query and we obtain a larger result set. Additional categories (which occur six times or more) can be found that are relevant to the enriched keywords. In this way we are

\footnotetext{
${ }^{6} \mathrm{https} / / /$ lucene.apache.org/solr/guide/6_6/the-dismax-query-parser.html

7 Added keywords ( $>2)$ : patient, cardiology, tavr, catheter ablation, dcb, ehra, esc area, esc member country, geographical esc region, lesion revascularization, non-european esc country, predictor, pulmonary hypertension, revascularization

Added categories (> 2): Cardiac \& Cardiovascular Systems, Health Care Sciences \& Services, Medicine, General $\&$ Internal, Peripheral Vascular Disease, Surgery
} 
able to obtain a profile which allows us to investigate the research activities of Leipzig University relevant to the topic reflected by the original search term.

Figure 1: Histograms of collected keyword and category information well suited for profiling of academic careers, faculties or research projects.
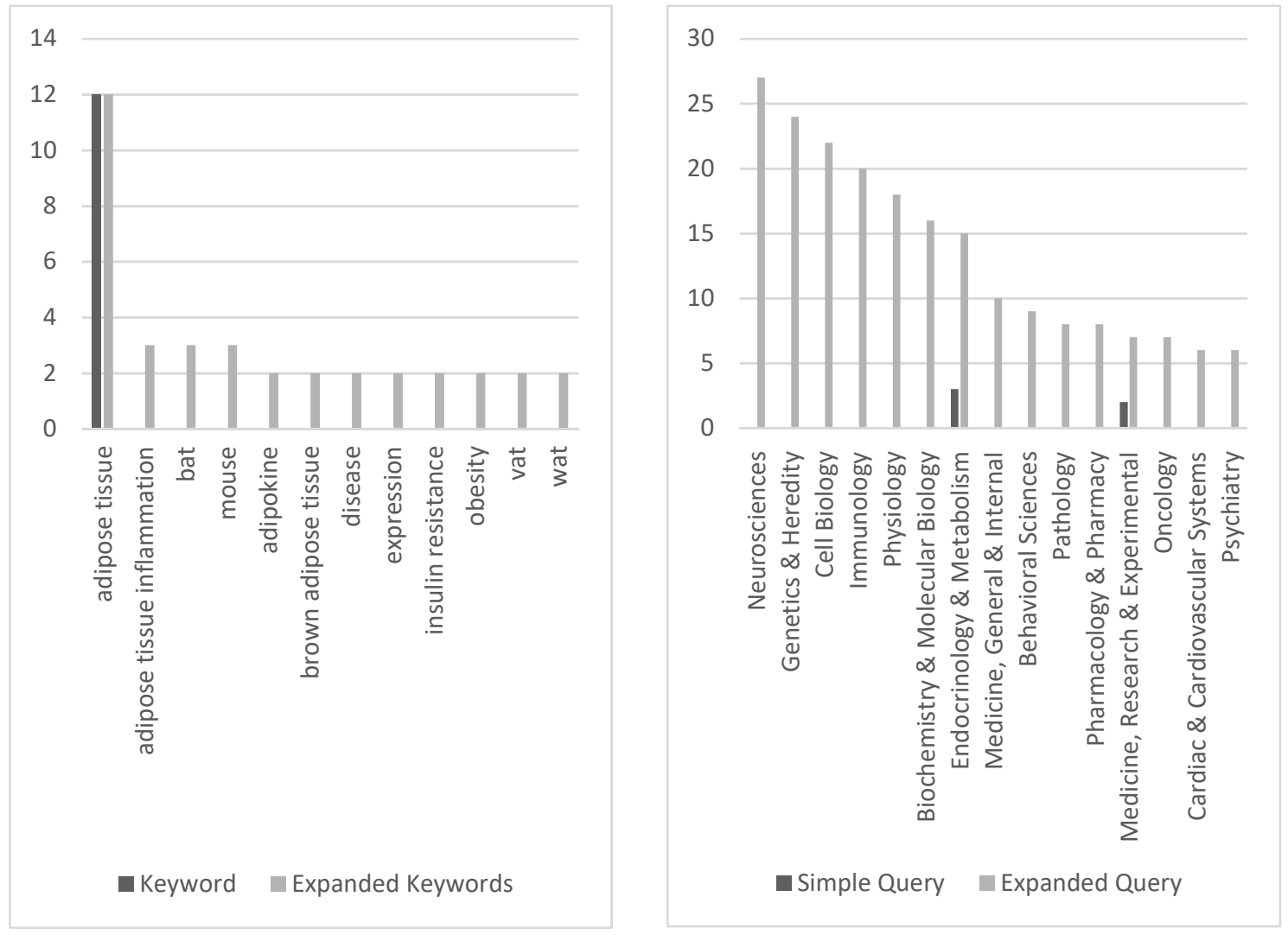

\section{Discussion}

In this work, we presented and evaluated several known algorithms for keyword extraction and text classification in scientific publications and project descriptions. In an example, we augmented the documents in a full-text index with the results of these algorithms to support the search process and to enable analysis applications based on the document repository, such as profiling authors or faculties. The evaluation demonstrates that graph-based keyword extraction approaches such as KECNW can compete with TF-IDF based algorithms with respect to precision, recall and F1 Score. However, the computing time, which is considerably longer than that of TF-IDF based approaches, has been noticed negatively. This can possibly be improved but was not pursued in this work. Furthermore, the candidates of TAKE gave better results than those of RAKE. We also found out that the score calculation of the candidates with average values achieved better results than with summation, since the average prevents the preference of longer keywords. Nevertheless, the individual term scoring of RAKE / TAKE was not able to keep up with the results of the other presented methods.

Keyword extraction methods that only set a single term as a keyword are not meaningful enough since authors frequently assign multi-term keywords. The candidates extracted by TAKE have greater accordance with the author keywords than those extracted by RAKE. However, many keywords that are not directly contained in the text are missing since the authors derive them from the text with the help of their own knowledge. However, since authors also assign keywords that do not appear directly in the text, a fixed vocabulary is required that can be assigned to texts. One such approach is Wikify (Mihalcea \& Csomai, 2007). Wikify uses the Wikipedia headings as a fixed vocabulary and assigns them to the texts. As an additional 
improvement, one could create a stopword list given a specific context such as publications and project descriptions. This can prevent unimportant terms from becoming too important, such as the word project in project descriptions, which usually describes all these texts.

To classify the texts, the Web of Science categories with the derivable CWTS Leiden categories where used as a taxonomy for English publications. For the German project descriptions, our experiments used the first three levels of the GEPRIS classifications as taxonomies. We applied a SVM in order to classify the documents. Since the texts of these domains cannot necessarily be assigned to a single label, we transferred the problem to binary relevance and created a single classifier for each label. Due to the resulting class imbalance, it is inevitable to use oversampling as the evaluation demonstrated. Furthermore, we implemented ensembles of classifier chains in order to include label hierarchies. This is particularly profitable with a great number of different labels. The classifications results are satisfactory. Only the Web of Science categories appear to be problematic with regard to their low F1 score. Due to the large amount of different labels, which may overlap thematically or could also be superordinate fields, it is difficult to determine an exact result. With the test data, it often happens that labels are awarded that do not match the correct label but are very close to it and one would think as an assessor that the label was assigned correctly. Another way to improve the classification is to create larger training datasets. Especially in the Web of Science dataset with 254 classes it is difficult to create a good classifier with only $\sim 200$ examples per label. Finally, it would be possible to use PU Learning (Sansone, De Natale, \& Zhou, 2018). PU means positive unlabeled. Although there are labels for all examples in the training and test datasets, it may be useful to consider the false class as unlabeled, because some examples from the false class may overlap with the labels of the positive class. For example, there are publications in the Web of Science that are only assigned to the class Cell Biology. However, there is also the class Biology, which is theoretically a superordinate field of cell biology. Since this is a class, which can be described with the same features as Cell Biology, it does not make any sense to train such a document as negative class example. PU Learning would consider this aspect.

Finally, we demonstrated the application of the extracted metadata in the context of search indexes and profiling. A search query expansion creates results that are additionally relevant for a search query. Furthermore, we can create categorical summaries of documents thanks to the metadata determined. The extracted metadata enables researchers to use keywords for finding other researchers working on the same topic. This aspect could promote cooperation between researchers who might not be aware of mutual research interests.

\section{References}

Aly, M. (2005). Survey on multiclass classification methods. Neural Netw, 19, 1-9.

Ariannezhad, M., Montazeralghaem, A., Zamani, H., \& Shakery, A. (2017). Iterative Estimation of Document Relevance Score for Pseudo-Relevance Feedback. In J. M. Jose, C. Hauff, I. S. Altıngovde, D. Song, D. Albakour, S. Watt, \& J. Tait (Eds.), Advances in Information Retrieval (pp. 676-683). Springer International Publishing.

Beliga, S., Meštrović, A., \& Martinčić-Ipšić, S. (2015). An overview of graph-based keyword extraction methods and approaches. Journal of Information and Organizational Sciences, 39(1), 1-20.

Bellaachia, A., \& Al-Dhelaan, M. (2012). Ne-rank: A novel graph-based keyphrase extraction in twitter. Proceedings of the The 2012 IEEE/WIC/ACM International Joint Conferences on Web Intelligence and Intelligent Agent Technology-Volume 01, 372-379. IEEE Computer Society.

Biswas, S., Bordoloi, M., \& Shreya, J. (2017). A Graph Based Keyword Extraction Model using Collective Node Weight. Expert Systems with Applications, 97. https://doi.org/10.1016/j.eswa.2017.12.025

Brück, T. vor der, Eger, S., \& Mehler, A. (2016). Complex Decomposition of the Negative Distance kernel. CoRR, abs/1601.00925. Retrieved from http://arxiv.org/abs/1601.00925

Dunning, T. (1993). Accurate methods for the statistics of surprise and coincidence. Computational Linguistics, 19(1), 61-74. 
Fan, R.-E., Chang, K.-W., Hsieh, C.-J., Wang, X.-R., \& Lin, C.-J. (2008). LIBLINEAR: A Library for Large Linear Classification. Journal of Machine Learning Research, 9, 1871-1874.

Honnibal, M., \& Montani, I. (2017). spacy 2: Natural language understanding with bloom embeddings, convolutional neural networks and incremental parsing. To Appear.

Hulth, A. (2003). Improved Automatic Keyword Extraction Given More Linguistic Knowledge. Proceedings of the 2003 Conference on Empirical Methods in Natural Language Processing, 216-223. https://doi.org/10.3115/1119355.1119383

Keikha, A., Ensan, F., \& Bagheri, E. (2017). Query expansion using pseudo relevance feedback on wikipedia. Journal of Intelligent Information Systems, 1-24.

Mihalcea, R., \& Csomai, A. (2007). Wikify!: Linking Documents to Encyclopedic Knowledge. Proceedings of the Sixteenth ACM Conference on Conference on Information and Knowledge Management, 233-242. https://doi.org/10.1145/1321440.1321475

Mihalcea, R., \& Tarau, P. (2004). Textrank: Bringing order into text. Proceedings of the 2004 Conference on Empirical Methods in Natural Language Processing.

Pay, T. (2016). Totally automated keyword extraction. Big Data (Big Data), 2016 IEEE International Conference On, 3859-3863. IEEE.

Rayson, P., Berridge, D., \& Francis, B. (2004). Extending the Cochran rule for the comparison of word frequencies between corpora. 7th International Conference on Statistical Analysis of Textual Data (JADT 2004), 926-936.

Read, J., Pfahringer, B., Holmes, G., \& Frank, E. (2009). Classifier Chains for Multi-label Classification. In W. Buntine, M. Grobelnik, D. Mladenić, \& J. Shawe-Taylor (Eds.), Machine Learning and Knowledge Discovery in Databases (pp. 254-269). Berlin, Heidelberg: Springer Berlin Heidelberg.

Read, J., Pfahringer, B., Holmes, G., \& Frank, E. (2011). Classifier chains for multi-label classification. Machine Learning, 85(3), 333. https://doi.org/10.1007/s10994-011-5256-5

Rose, S., Engel, D., Cramer, N., \& Cowley, W. (2010). Automatic keyword extraction from individual documents. Text Mining: Applications and Theory, 1-20.

Sansone, E., De Natale, F. G., \& Zhou, Z.-H. (2018). Efficient training for positive unlabeled learning. IEEE Transactions on Pattern Analysis Annotationd Machine Intelligence.

Singhal, A., Kasturi, R., \& Srivastava, J. (2013). Automating document annotation using open source knowledge. Web Intelligence (WI) and Intelligent Agent Technologies (IAT), 2013 IEEE/WIC/ACM International Joint Conferences On, 1, 199-204. IEEE.

Sjögårde, P., \& Ahlgren, P. (2019). Granularity of algorithmically constructed publication-level classifications of research publications: Identification of specialties. ArXiv:1901.05273 [Cs]. Retrieved from http://arxiv.org/abs/1901.05273

Sparck Jones, K. (1972). A statistical interpretation of term specificity and its application in retrieval. Journal of Documentation, 28(1), 11-21. https://doi.org/10.1108/eb026526

Uslu, T., Mehler, A., Niekler, A., \& Baumartz, D. (2018). Towards a DDC-based Topic Network Model of Wikipedia.

Uwe Quasthoff, D. G., \& Eckart, T. (2015). Building Large Resources for Text Mining: The Leipzig Corpora Collection. Springer.

Waltinger, U., Mehler, A., Lösch, M., \& Horstmann, W. (2009). Hierarchical Classification of OAI Metadata Using the DDC Taxonomy. NLP4DL/AT4DL, 29-40.

Wolfram, D. (2015). The symbiotic relationship between information retrieval and informetrics. Scientometrics, 102(3), 2201-2214. https://doi.org/10.1007/s11192-014-1479-0

$\mathrm{Xu}$, J., \& Croft, W. B. (1996). Query expansion using local and global document analysis. Proceedings of the 19th Annual International ACM SIGIR Conference on Research and Development in Information Retrieval, 4-11. ACM. 